\title{
Overall and local contrast in multiple schedules: Effects of stimulus similarity and discrimination performance
}

\author{
PATRICIA M. BLOUGH \\ Brown University, Providence, Rhode Island
}

\begin{abstract}
An experiment with pigeons related overall and local behavioral contrast to similarity between stimuli signaling multiple-schedule components. Similarity was defined both physically and by discrimination performance. Initial and final baseline conditions used two equal random-interval schedules. During two intervening test periods, the schedule accompanying one component was changed to extinction. In the first test, components alternated strictly; in the second test, ran. dom component sequences were used. Signaling wavelength stimuli were separated by $1.5,2$, or $14 \mathrm{~nm}$. Overall positive contrast occurred reliably, but its amount depended neither on wavelength difference nor on discrimination performance. Local positive contrast was less fre quently observed when signaling stimuli were physically dissimilar; however, the effect was most closely related to actual discrimination performance. The relationship between discrimination and local contrast was nonmonotonic, indicating maximum local contrast at intermediate dis. criminations.
\end{abstract}

Extensive research and theory have addressed behavioral contrast in multiple schedules (see review by Williams, 1983). In efforts to understand this phenomenon, researchers have distinguished between overall and local forms. Overall positive contrast is defined as an increase in response rate during a constant schedule component, along with a reduction in reinforcement rate during alternative components. Local contrast has been assessed in two ways, one that considers between-component sequences and one that considers within-component patterning. Between-component local contrast is positive if overall response rate during a given component is relatively high when the immediately preceding component had been associated with a relatively low reinforcement rate. Within-component local contrast is positive when response rate is elevated during the initial portion of a relatively rich component, yielding a decreasing within-component pattern. Negative overall and local contrast have similar forms, but in opposite directions.

Although overall and local contrast are conceptually related, the two forms may not always coexist. For example, local contrast usually diminishes with exposure to unequal schedules (e.g., Nevin \& Shettleworth, 1966), but overall contrast does not. In his review, Williams (1983) pointed to the transitory nature of local contrast as a major feature that distinguishes local from overall contrast. In this and subsequent papers (Williams, 1988;

This research was supported by Grant BNS 80-25515 from the National Science Foundation. I thank Stephen R. Blough for his help with the regression analyses and Donald $\mathrm{S}$. Blough for his suggestions concerning the manuscript. The author's address is Department of Psychology, Brown University, Providence, RI 02912.
Williams \& Wixted, 1986), Williams reasoned that withincomponent local contrast accounts for overall contrast during early exposure to unequal schedules. However, as the local form dissipates, the overall form persists because of an anticipatory effect that emerges as exposure continues. This effect reflects control by the schedule during the following component: Within-component rates follow a rising pattern when the following schedule is to be relatively lean; they drop when the following schedule is to be relatively rich (see also Buck, Rothstein, \& Williams, 1975). Applied to positive contrast, then, this analysis would attribute overall contrast initially to local response rate elevations at rich component onsets. After further exposure, however, the overall rate elevation would be attributable to anticipatory rate increases, which are most marked near the end of a component. According to Williams's formulation, anticipatory effect exerts stronger influence on overall contrast than does local contrast.

Another way to consider the difference between local and overall contrast is in terms of discrimination performance, whose improvement is typically associated with the decline of local but not overall contrast. However, discrimination performance depends not only on exposure, but also on physical stimulus similarity. Thus, similarity may be included among the conditions that affect the two forms of contrast differently. Although research addressing stimulus factors is sparse, several researchers have considered this issue.

Some evidence indicates that overall contrast is, in fact, favored by dissimilar signaling stimuli. Mackintosh, Little, and Lord (1972) reported greater positive contrast when schedule components were signaled by line-tilt stimuli that differed by $90^{\circ}$ than when these stimuli differed by $15^{\circ}$. Williams (1988) compared intra- and in- 
terdimensional stimulus pairs in a study that emphasized the direction of within-component interactions. Anticipatory effects, defined as appropriate changes in withincomponent response patterns, were more pronounced when the signaling stimuli were most different. Williams concluded that stimulus dissimilarity favored overall contrast.

If relatively poor discrimination performance favors local contrast, stimulus similarity should enhance this form. However, the current picture is inconsistent. In a study that varied the similarity of signaling stimulus wavelengths, P. M. Blough (1983) found fairly clear evidence for stronger local contrast with increasing stimulus similarity; however, this finding was significant for the between-component measure, and not for the withincomponent measure. Malone (1976) explored this issue in an experiment that used multiple line-tilt stimuli and concluded that stimulus similarity diminished withincomponent contrast. In his comparison between intra- and interdimensional stimulus pairs, Williams (1988) did not obtain reliable within-component local contrast.

There are a number of good reasons why the similarity-contrast relationship is still unclear. One is that a wide range of stimulus similarity is difficult to achieve, especially for pigeons, whose excellent vision may lead to ceiling effects when standard visual stimuli are used. Furthermore, individual differences in the rate and extent of discrimination learning may obscure group effects based on physical stimulus values. Finally, if local contrast is indeed enhanced by stimulus similarity, there must be a limit to this effect, for it should not occur when the stimuli are so similar that they cannot be discriminated. Indeed, a function reported by P. M. Blough (1983, Figure 4) suggests a nonmonotonic relationship between similarity and discrimination performance.

The present study defined more completely the relation between discrimination and positive contrast for both overall and local forms. It was designed to dissociate training effects from effects of physical stimulus similarity. Signaling stimuli were selected to yield a wide range of steady-state discrimination performances, so that their role could be explored quantitatively. An additional manipulation was the order in which schedule components occurred. One condition used the strictly alternating sequences typical of related work; however, this procedure did not permit assessment of between-component local contrast. Thus, a second condition used random component sequences so that rates in a constant schedule could be compared according to the schedule in the immediately preceding component. Comparisons between alternating and random sequences also addressed Williams's (1983) following-schedule account of overall contrast. By removing sequential cues, the random condition degraded information about the schedule in the following component. According to Williams's account, this manipulation should diminish overall contrast.

\section{METHOD}

\section{Subjects}

Eighteen White Carneau pigeons participated. Fourteen had served in an undergraduate laboratory course, in which they were exposed briefly to a variety of procedures; 2 birds had learned discriminations among form stimuli; and 2 were experimentally naive. Six pigeons were assigned to each of three stimulus-range groups; both naive birds were members of Group 3; 1 of the more experienced birds was assigned to Group 2 and 1 to Group 3 . The subjects were run in squads of 3 . All birds were maintained at $80 \%$ of their freefeeding weights.

\section{Apparatus}

The apparatus is more fully described by P. M. Blough (1983). Briefly, there were three subject boxes located in a room separate from the controlling equipment. Each contained a fan and a loudspeaker through which white noise was delivered. Mounted on the front panels of the subject chambers were a response key and, below that, an opening to a food hopper. Stimuli were provided by an optical system, which consisted of a tungsten source, a grating monochromator, and a neutral density wedge. An image of the monochromator's grating was focused on fiber optics cables, which led to the backs of the clear response keys. Operation of the keys yielded an audible click. A Digital Equipment Corporation PDP$11 \mathrm{~V} 03$ computer provided on-line control.

\section{Procedure}

After magazine training, the subjects learned to peck the key, illuminated by a $600-\mathrm{nm}$ light, through autoshaping. After 60 reinforced responses, exposure to the multiple schedule began. During initial sessions, reinforcement was programmed with a gradually decreasing probability until the birds responded regularly on the standard random-interval (RI) 60-sec schedule. Regular experimental sessions then began.

Each session consisted of a series of trials, made up of multipleschedule components, $60 \mathrm{sec}$ in duration and separated by 2 -sec intertrial intervals (ITIs). During each ITI, the houselight and keylight were unlit. One signaling wavelength was al ways $600 \mathrm{~nm}$. The wavelength signaling the alternate component varied among groups. For Group 1, it was $598.5 \mathrm{~nm}$ for 3 birds and $601.5 \mathrm{~nm}$ for the other 3; for Group 2, it was always $598 \mathrm{~nm}$; and for Group 3, it was always $586 \mathrm{~nm}$. The constant component was accompanied by the longer wavelength for half the birds in each condition and the shorter wavelength for the other half. Energy was adjusted so that the lights were equivalent in luminance for the pigeon eye (D. S. Blough, 1957).

There were three experimental conditions, always occurring in the same sequence. During an initial baseline condition, sessions consisted of 60 trials, all accompanied by the RI 60-sec reinforcement schedule and signaled by the assigned wavelengths in a strictly alternating sequence. Testing during an alternating RI/Ext condition then began; that is, the schedule of reinforcement for one component changed to extinction, and this component alternated strictly with the unchanged component. Sessions consisted of 80 such trials. The third condition used the same schedules, but the components occurred in random sequence. A random block design selected the components, ensuring that an equal number of each occurred in a session and that sequences of a given component were not longer than four. Sessions consisted of 80 trials. The experiment ended with a return to the baseline condition, with components again occurring in strictly alternating sequence. (It should be noted that, for Group 1, wavelength separation was $1 \mathrm{~nm}$ during the initial baseline condition and the early portion of the second condition. Since most birds did not appear to be learning the discrimination, the sepa- 
Table 1

Steady-State Discrimination Data Averaged Over Final Nine Sessions for Each Condition and Over the 6 Birds Constituting a Stimulus-Range Group

\begin{tabular}{|c|c|c|c|c|c|c|c|c|}
\hline \multirow[b]{2}{*}{ Group } & \multicolumn{2}{|c|}{ Baseline } & \multicolumn{2}{|c|}{$\begin{array}{l}\text { Alternating } \\
\text { Sequence }\end{array}$} & \multicolumn{2}{|c|}{$\begin{array}{l}\text { Random } \\
\text { Sequence }\end{array}$} & \multicolumn{2}{|c|}{ Baseline } \\
\hline & $M$ & $S D$ & $M$ & $S D$ & $M$ & $S D$ & $M$ & $S D$ \\
\hline 1 & .50 & .01 & .72 & .11 & .77 & .07 & .54 & .05 \\
\hline 2 & .49 & .01 & .80 & .06 & .83 & .06 & .52 & .01 \\
\hline 3 & .47 & .02 & .94 & .05 & .97 & .03 & .48 & .03 \\
\hline
\end{tabular}

Note $-M=$ geometric mean [(rate in $S+) /($ rate in $S++$ rate in $S-)]$.

ration was increased to $1.5 \mathrm{~nm}$, and evaluation for trend was restarted.)

Each phase ended when all members of a 3-bird squad met a stability criterion for trend (P. M. Blough, 1983; Spealman \& Gollub, 1974). This rule required a minimum of 14 sessions, terminating in 9 sessions during which a performance measure neither increased nor decreased systematically. The performance measure, preselected as that most likely to change systematically during a condition, was response rate during initial baseline. During subsequent phases, the stability criterion was applied to the discrimination index (DI). The number of sessions required to meet the criterion varied most notably during the first testing condition: Group 1 required 31 to 33 sessions, Group 2 required 21 to 27 sessions, and Group 3 required 15 sessions.

\section{RESULTS}

The following analyses focus on positive overall and local contrast; response rates in extinction were too low to assess negative local contrast. Effects based on physical stimulus differences are considered in the first section below; a second section evaluates effects based on individual differences in discrimination performance.

\section{Effects of Wavelength Difference}

Tables 1-3 show steady-state means. The values, based on the final sessions that met the criterion for trend, summarize the findings for each group in each condition. Statistical evaluations that compared baseline measures with those obtained during RI/Ext testing used averages over initial and final RI/RI baselines. Effects described as nonsignificant were associated with a $p$ value greater than .05 .

Table 1 summarizes discrimination performance, described by a discrimination index (DI, the number of responses during the constant components divided by the total number of responses overall). All birds learned the required discriminations; that is, in every case the DI increased during the unequal schedule conditions and returned to near chance values during the final baseline phase. As expected, larger DIs were associated with larger wavelength differences, and the outcome of a one-way analysis of variance (ANOVA) established the significance of this group effect. For the alternating condition, $F(2,15)$ $=11.20, p<.001 ;$ for the random condition, $F(2,15)$ $=22.15, p<.0001$. It is noteworthy that discrimination was slightly better during the random component sequence; thus, removal of sequential cues did not diminish discriminability.

Table 2 summarizes overall positive contrast, which would appear as higher constant-component rates during the RI/Ext conditions than during the averaged baseline phases. This effect was seen in all groups, although it was diminished by the failure of final baseline rates to return to their initial values. The largest amount of contrast occurred in Group 2, associated with the intermediate stimulus difference. Mixed-model ANOVAs evaluated the effects of schedule change and wavelength difference. Comparing baseline to the alternating RI/Ext condition, there was a significant effect of schedule change $[F(1,15)$ $=9.0, p<.01]$, but there were no significant effects of stimulus difference and no significant interaction. Comparing baseline with the random RI/Ext condition, there was also a significant effect of schedule change $[F(1,15)$ $=16.9, p<.01]$, but again there was no significant effect of wavelength difference and no significant interaction. Thus, although overall contrast was established, the findings did not reveal an effect of stimulus difference.

The amount of overall contrast in the random conditions was slightly higher than it was in the alternating condition. For the combined groups, this difference, evaluated by a $t$ test, was not significant.

Table 2

Steady-State Rate Data Averaged over Final Nine Sessions for Each Condition and Over the 6 Birds Constituting a Stimulus-Range Group

\begin{tabular}{|c|c|c|c|c|c|c|c|c|c|c|}
\hline \multirow[b]{4}{*}{ Group } & \multicolumn{10}{|c|}{ Testing } \\
\hline & \multirow{2}{*}{\multicolumn{2}{|c|}{ Baseline }} & \multirow{2}{*}{\multicolumn{2}{|c|}{$\begin{array}{c}\text { Alternating } \\
\text { Sequence }\end{array}$}} & \multicolumn{6}{|c|}{ Random Sequence } \\
\hline & & & & & \multicolumn{2}{|c|}{ F/RI 60-sec } & \multicolumn{2}{|c|}{ F/Ext } & \multicolumn{2}{|c|}{ Baseline } \\
\hline & $M$ & $\overline{S D}$ & $\bar{M}$ & $S D$ & $M$ & $S D$ & $M$ & $S D$ & $M$ & $S D$ \\
\hline 1 & 70 & 27 & 95 & 47 & 105 & 46 & 110 & 51 & 92 & 44 \\
\hline 2 & 79 & 34 & 170 & 120 & 170 & 117 & 175 & 124 & 108 & 60 \\
\hline 3 & 69 & 32 & 114 & 44 & 131 & 53 & 126 & 51 & 110 & 52 \\
\hline
\end{tabular}

Note $-F=$ following. $M=$ arithmetic mean; responses per minute. 
Table 3

Steady-State Within-Component Ratios Averaged Over Final Nine Sessions for Each Condition and Over the 6 Birds Constituting a Stimulus-Range Group

\begin{tabular}{|c|c|c|c|c|c|c|c|c|c|c|}
\hline \multirow[b]{4}{*}{ Group } & \multicolumn{10}{|c|}{ Testing } \\
\hline & \multirow{2}{*}{\multicolumn{2}{|c|}{ Baseline }} & \multirow{2}{*}{\multicolumn{2}{|c|}{$\begin{array}{c}\text { Alternating } \\
\text { Sequence }\end{array}$}} & \multicolumn{6}{|c|}{ Random Sequence } \\
\hline & & & & & \multicolumn{2}{|c|}{$\mathrm{F} / \mathrm{RI} 60$-sec } & \multicolumn{2}{|c|}{ F/Ext } & \multicolumn{2}{|c|}{ Baseline } \\
\hline & $M$ & $S D$ & $M$ & $S D$ & $M$ & $S D$ & $M$ & $S D$ & $M$ & $S D$ \\
\hline 1 & 1.02 & .04 & 1.00 & .07 & 1.06 & .07 & 1.08 & .07 & 1.03 & .06 \\
\hline 2 & 1.02 & .04 & 1.04 & .07 & 1.05 & .04 & 1.06 & .06 & 1.02 & .08 \\
\hline 3 & 1.01 & .04 & .92 & .10 & 1.00 & .01 & 1.00 & .08 & 1.01 & .04 \\
\hline
\end{tabular}

Table 3 summarizes local contrast in its withincomponent form. This effect would appear as declining rate patterns during the constant component of the RI/Ext conditions. The within-component pattern was quantified by a ratio that compared rate in the first half with that in the last half of the appropriate 1-min components. Values exceeding 1.0 indicate the declining patterns that define local contrast. As seen in Table 3, such patterns were more marked in Groups 1 and 2 and in the random RI/Ext condition; of the 18 subjects, 17 showed higher within-component ratios in the random (F/Ext) than in the alternating condition. The exceptional bird was a member of Group 3. The hypothesis that the within-component ratios were equal to 1.0 was evaluated by $t$ test. This hypothesis was not rejected for any group in the alternating condition, but significant effects were seen in the random condition. In components preceded by extinction, local contrast was significant for Group $1[t(5)=2.79$, $p<.05$ ], and the outcome approached significance for Group $2[t(5)=2.44, p=.06]$. In components preceded by the constant schedule, local contrast was significant for Group $2[t(5)=2.92, p<.05]$, and the outcome approached significance for Group $1[t(5)=2.21, p=.08]$. One-way ANOVAs evaluated the effect of stimulus similarity on the strength of these patterns, but the outcome was not significant for either sequence. Thus, within-component local contrast was established when component sequences alternated randomly. Stimulus similarity favored its occurrence, but did not have a significant effect on its strength.

Local contrast, in its between-component form, would appear as relatively high response rates in components preceded by extinction. The random RI/Ext condition permitted this comparison. As seen in Table 2, rates for Groups 1 and 2 differed in the manner associated with local contrast; that is, they were relatively high following extinction. However, for Group 3, the relationship reversed; that is, rates were relatively low following extinction. Analyses employed a ratio between the two rates, and $t$ tests evaluated the hypothesis that the ratio was equal to 1.0. This hypothesis was rejected in the case of Group 3 $[t(5)=-3.63, p=.01]$ and approached significance in the case of Group $1[t(5)=2.07, p=.09]$. A one-way ANOVA evaluated the effect of stimulus similarity on the strength of the between-component ratio. The outcome was significant $[F(2,15)=6.31, p=.01]$. Thus, this form of local contrast was established only marginally in the high-similarity group, and an opposite effect appeared when stimuli were most dissimilar. The strength of the between-component effect depended on the difference between the signaling wavelengths.

\section{Effects of Discrimination Performance}

As shown in Table 1, mean DI had the expected monotonic relationship to stimulus similarity. Nonetheless, there remained a good deal of variability among individuals trained with given wavelength pairs. Further analysis was guided by the hypothesis that performance, rather than physical definitions of similarity, would improve our understanding of contrast. These analyses used both the DI and extinction responding as performance measures. Their relation to contrast was explored with the aid of regression analyses prepared by the SAS Institute (Freund \& Littell, 1981). Functions fit to the data below employed a quadratic model. The value $r^{2}$ describes the goodness of fit in terms of variance accounted for. The significance of this value was evaluated by an $F$ test, addressing the null hypothesis that both the linear and the quadratic coefficients were equal to zero. Reported $t$ values addressed the following null hypotheses: (1) that the coefficient associated with the best fitting linear equation was equal to zero, and (2) that, controlling for the linear contribution, the coefficient associated with an added quadratic factor was equal to zero.

Figure 1 describes the relationship between overall contrast and discrimination. Although nonmonotonic functions provided the best fits for both alternating and random conditions, in neither case did the model account for a significant amount of the variance. Thus, the evidence did not document a relation between overall contrast and discrimination.

Figure 2 describes the relationship between withincomponent patterning and discrimination. In these cases, fits were considerably better. Most striking are the data for the alternating condition (top panel), where the quadratic model accounted for a significant amount of the variance $\left(r^{2}=.63 ; F=12.70, p<.001\right)$. The coefficient associated with the linear factor alone was significant $(t=2.70, p<.05)$, and the quadratic factor contributed significantly to the fit $(t=-3.57, p<.01)$. Thus, both limbs of this nonmonotonic function contributed significantly to an account of the variance. Max- 


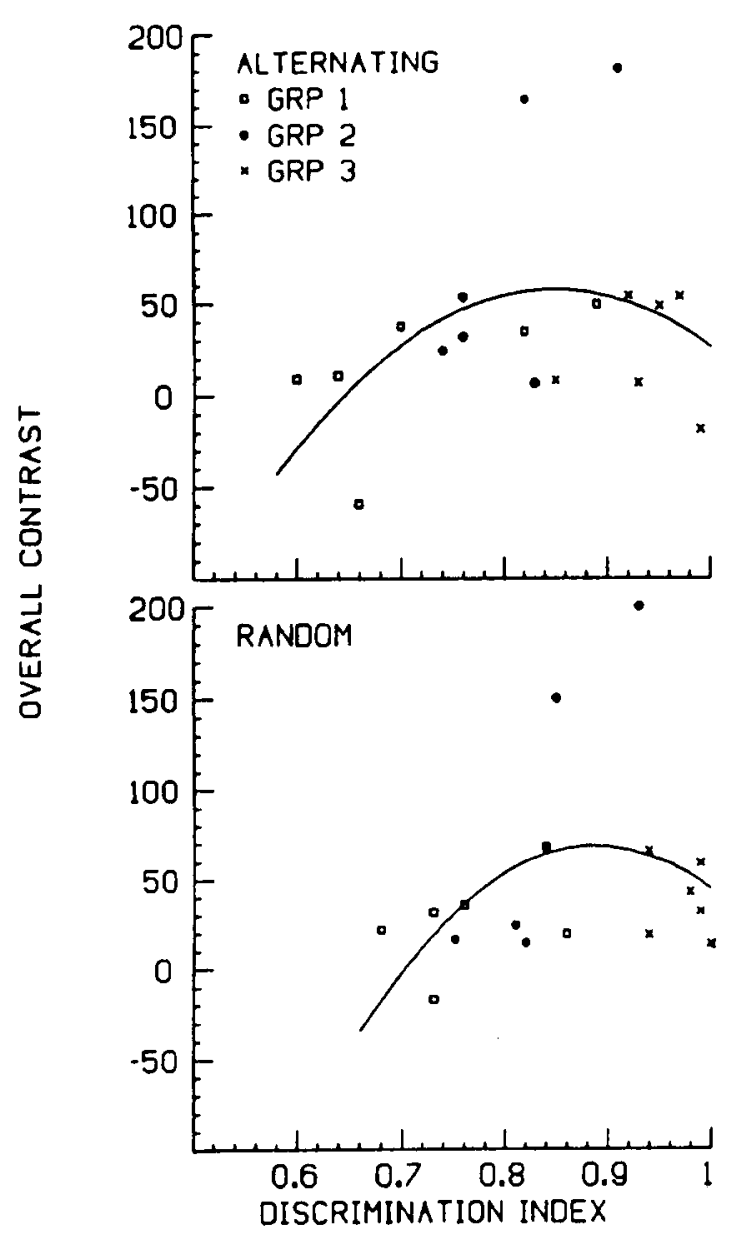

Figure 1. The relation between contrast and discrimination performance. Contrast is determined from rates during a constant random interval (RI) 60-sec component; it is quantified as the difference between rates during multiple random-interval/extinction (Mult RI/Ext) and Mult RI/RI. The discrimination index is a ratio between number of responses during $\mathbf{R I}$ and overall number of responses during the RI/Ext schedule. The top panel refers to a condition in which schedule components strictly alternated. The bottom panel refers to a condition in which component sequences were random. For Groups 1, 2, and 3, denoted by different symbols, the wavelength separation between component-signaling stimuli was $1.5,2$, and $14 \mathrm{~nm}$, respectively. Points refer to individual birds. The function is drawn according to the best fitting quadratic equation.

imum local contrast, defined by the maximum of the fitted quadratic function, was associated with a DI of .75.

For the random condition, in components preceded by extinction (center panel), the quadratic model also accounted for a significant amount of the variance $\left(r^{2}=.49 ; F=7.22, p<.01\right)$. The contribution of the linear factor alone was significant $(t=3.04, p<.01)$, but the added contribution of the quadratic factor was not significant. It should be noted that, in this condition, DIs extended over a narrower range, leaving less room for local contrast to diminish with poor discriminability. The function's maximum (DI $=.78$ ) is close to that associated with the alternating condition.

For the random condition, in components preceded by equivalent schedules (bottom panel), the fit was less good and did not account for a significant amount of the variance. However, the contribution of the linear factor alone approached significance $(t=2.05, p=.06)$. Taken together, the data in Figure 2 provide strong evidence for a decline in local contrast as discrimination performance improves from a moderate to a high level. The decline associated with poor discriminations was marked for the alternating condition, and it seems reasonable to expect replication in random conditions that include sufficient instances of low DIs.

Figure 3 (left panel) describes the relation between discrimination performance and local contrast assessed by the between-component measure. The fit supplied by the regression analysis, although relatively poor $\left(r^{2}=.32\right)$, approached significance $(F=3.48, p=.06)$. Furthermore, there was a significant contribution from the linear factor alone $(t=2.26, p<.05)$. By this measure, peak contrast was again associated with a DI of .78. Thus, local contrast, defined by the between-component measure, also appeared to be strongest at intermediate levels of discrimination.

An alternative measure of discrimination performance is the rate of responding within extinction components. Figure 3 (right panel) relates between-component contrast to rate in extinction, establishing a better fit $\left(r^{2}=.52\right.$; $F=8.15, p<.01$ ). As seen on the graph, the quadratic factor made no contribution, but the linear factor alone was significant $(t=4.17, p<.001)$. This case was the only one in which extinction responding yielded a markedly different correlational pattern. For withincomponent measures, analyses based on extinction responding provided similar increasing patterns, but less good fits to the data. Rate in extinction and the DI are conceptually related, and in this study had a moderately strong association $(r=-.65$ and -.76 for the alternating and random conditions, respectively). It is unclear why extinction responding accounted so much better for the variance in the between-component form of local contrast.

Figure 4 addresses the transiency of local contrast. These data follow changes in the within-component measure, along with corresponding changes in the DI during the alternating RI/Ext condition. For reference, each bird's mean within-component ratio during initial baseline is also shown. These birds made up Group 3, the wide-range group; Birds 515 and 721 were the experimentally naive subjects. All birds showed evidence of transient local contrast; that is, early in the birds' exposure to this condition, the within-component ratio rose above 1.0 and above its baseline value. Furthermore, the associated functions tended to decline, although there were individual differences in the rate and in the amount of change that took place.

\section{DISCUSSION}

The present findings shed new light on relationships between multiple-schedule contrast and the discriminability between stimuli signaling the components. Evaluations based on a physical definition of similarity revealed in- 

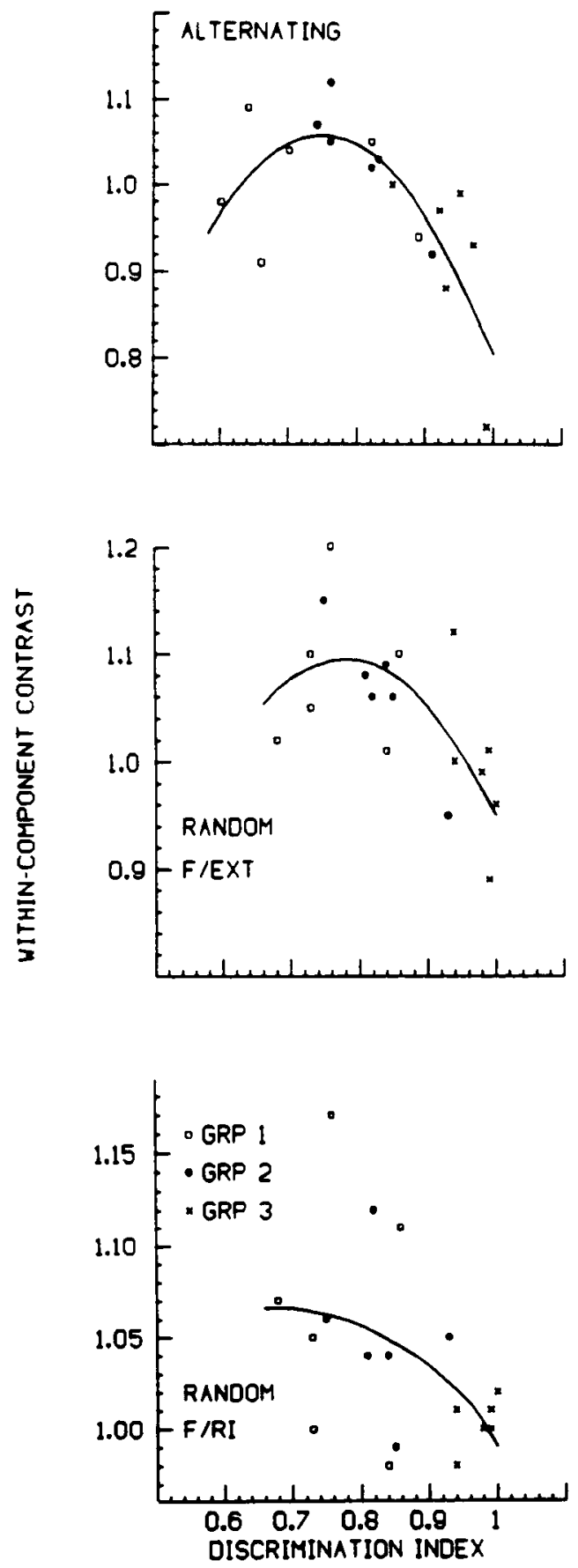

Figure 2. The relation between within-component (WC) local contrast and discrimination. Contrast is quantified as the ratio between rates in the first and last halves of the constant component. The top panel refers to the condition in which schedule components strictly alternated. The middle panel refers to the condition in which sequences were random and includes data only for random-interval (RI) components that followed extinction. The bottom panel also refers to the random condition, but includes data only for RI components that followed RI components of equivalent value. For Groups 1, 2, and 3, denoted by different symbols, the wavelength separation between component-signaling stimuli was $1.5,2$, and $14 \mathrm{~nm}$, respectively. Points refer to individual birds. The function is drawn according to the best fitting quadratic equation. stances in which stimulus similarity seemed to favor local contrast; however, the collective evidence was of marginal significance. Evaluations based on discrimination performance, however, clarified these relationships, indicating strongest local contrast at intermediate discrimination levels. Overall contrast was considerably more robust, occurring in almost all birds over a wide range of stimulus differences and discrimination performances.

Local contrast tends to be difficult to capture experimentally, and studies of stimulus factors have yielded conflicting outcomes. Given the importance of individual differences in discrimination performance, this variability is not surprising. The present findings (Tables 2 and 3) are consistent with previous research that found greater local contrast associated with smaller stimulus differences (P. M. Blough, 1983). They also are consistent with reports that note the transience of this effect (Bloomfield, 1966; Nevin \& Shettleworth, 1966) when discriminations are well learned (Figure 4). Furthermore, the outcome seems related to results from multiple-schedule experiments that show rate increases following the introduction of new components signaled by new and presumably poorly discriminated values (Catania \& Gill, 1964; Malone, 1976).

Varying definitions of local contrast also may account for inconsistencies among studies and for the fragility of the effect. Ideally, for example, within-component local contrast, like the overall form, should be assessed with reference to a baseline condition (McSweeney \& Norman, 1979). However, in the present study, some of the baseline data revealed rate elevations at component onsets. Although these effects were not strong enough to yield within-component ratios significantly different from 1.0, baseline was not an effective reference condition. Thus, as in Williams's (1988) study, local contrast in the present report is defined in terms of patterns obtained within experimental conditions. A second source of confusion occurs when within-component and between-component definitions are poorly distinguished. As noted in P. M. Blough (1983), these two measures are not necessarily equivalent.

An additional issue in measurement concerns the use of the parameter $a$, which describes the sensitivity of response ratios to reinforcement ratios (Williams, 1988). This measure makes intuitive sense, because the dependence between the two ratios accounts for overall contrast (Herrnstein, 1970). However, $a$ is a highly derived value that combines information about rates in both multiple-schedule components. Furthermore, as seen in Williams (1988), local effects based on $a$ differ from those based on rates during the unchanged component. This point is important because White, Pipe, and McLean (1984) found that $a$ increased with increasing dissimilarity among signaling stimuli; moreover, this effect was most pronounced at component onset. According to Williams's (1988) interpretation, the latter outcome supplies 


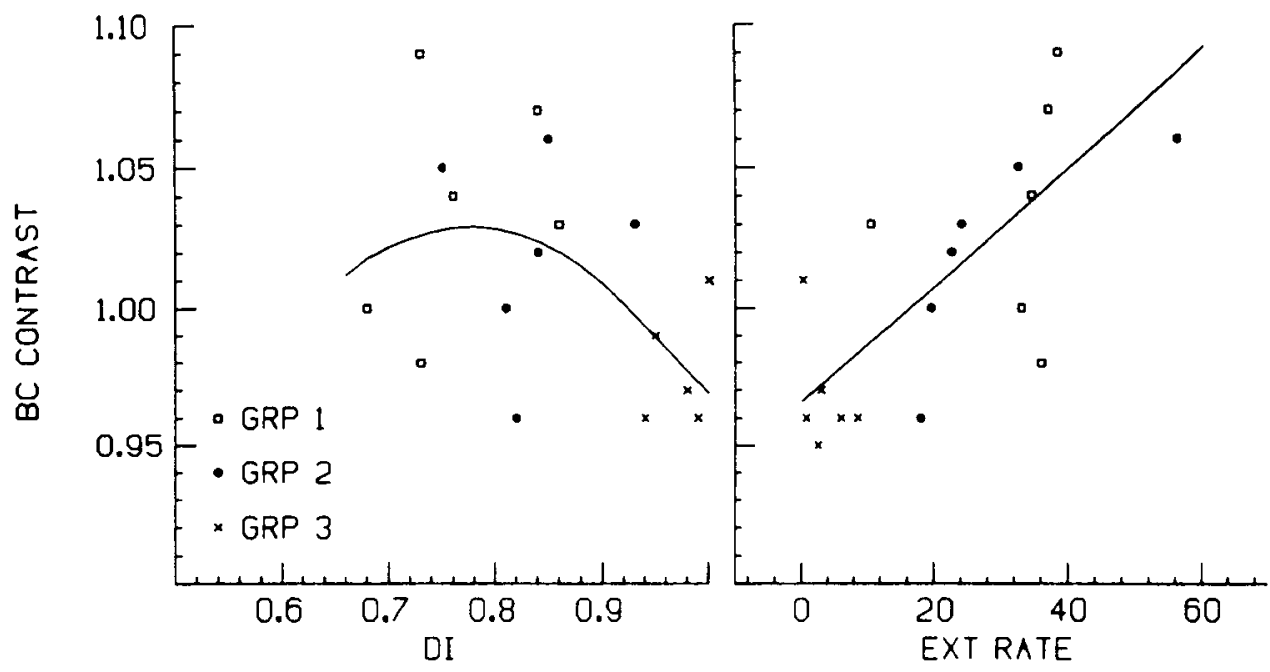

Figure 3. The relation between between-component (BC) local contrast and discrimination. Contrast is quantified as the ratio between random interval (RI) rates following extinction and RI rates following equivalent RI schedules. In the left panel, the discrimination ratio is the measure of discrimination (DI) performance; in the right panel, rate in the extinction (EXT) component is the measure. For Groups 1, 2, and 3, denoted by different symbols, the wavelength separation between component-signaling stimuli was $1.5,2$, and $14 \mathrm{~nm}$, respectively. Points refer to individual birds. The function is drawn according to the best fitting quadratic equation.

evidence for an increasing relationship between local contrast and stimulus dissimilarity. In fact, there are interesting asymmetries in White et al.'s early rate data (their Figure 2): Rate in the leaner schedule components declined sharply with stimulus dissimilarity, whereas rate in the richer components tended to be highest at intermediate similarity values. The $a$ parameter confounds these two different relationships.

In their assessment of stimulus factors, White et al. (1984) presented their several stimuli within each experimental session. This design raises the issue of dimensional contrast, whose relation to local and overall contrast is still unclear (P. M. Blough \& D. S. Blough, 1985). Positive dimensional contrast is exemplified in designs that use a stimulus continuum divided so that its halves signal different reinforcement rates. The effect is seen as relatively high rates associated with positive stimuli that have moderate physical distances from the values associated with the leaner schedule (P. M. Blough \& D. S. Blough, 1985; Catania \& Gill, 1964; White et al., 1984). It is strongest early in a schedule component (D. S. Blough, 1975; White et al., 1984). Thus, both local and dimensional contrast are favored by intermediate stimulus discriminability and factors that accompany a component's onset. The present data strengthen, but qualify Malone and Rowe's (1981) local contrast explanation for dimensional contrast; the present results indicate that the members of sequences involving moderate discriminability are likely to show the most contrast. Nonetheless, other types of stimulus interactions also may contribute to dimensional contrast (D. S. Blough, 1975), and it is possible that such interactions confound the assessment of local contrast by multiple-stimulus designs.
Accounts such as Malone and Rowe's (1981) emphasize the role of transitions between unequal schedule components. The present data raise questions about the importance of specific transitions. If the rate elevation defining positive within-component contrast is attributed specifically to the onset of a rich segment of a lean-rich schedule sequence, it should have been restricted to such transitions. However, in the present random condition, there was evidence for such elevations in components that followed equally valued schedules. Furthermore, although the data were more variable, contrast in such components had a nearly significant relationship with discrimination (Figure 2, bottom panel). Finally, there was a significant correlation of moderate size $(r=.52)$ between contrast in the two types of sequences. In a more extensive analysis of sequential factors, P. M. Blough and D. S. Blough (1985) concluded that dimensional contrast did not depend on sequential effects.

On the other hand, between-component local contrast is defined in sequential terms. In the present study, statistical documentation of this form was surprisingly weak; in fact, the strongest evidence was for the opposite effect, seen when stimuli were dissimilar. However, a number of birds did show between-component contrast (Figure 3), and the regression analyses suggest that the variance was best explained by extinction rates. In related work, however, P. M. Blough (1983) showed a fairly clear relationship between this form of contrast and the DI. Taken together, these experiments indicate that response rates depend on the value of the preceding schedule in a manner that depends on discriminability. Thus, sequential effects seem to enhance local contrast, but they are not necessary. 


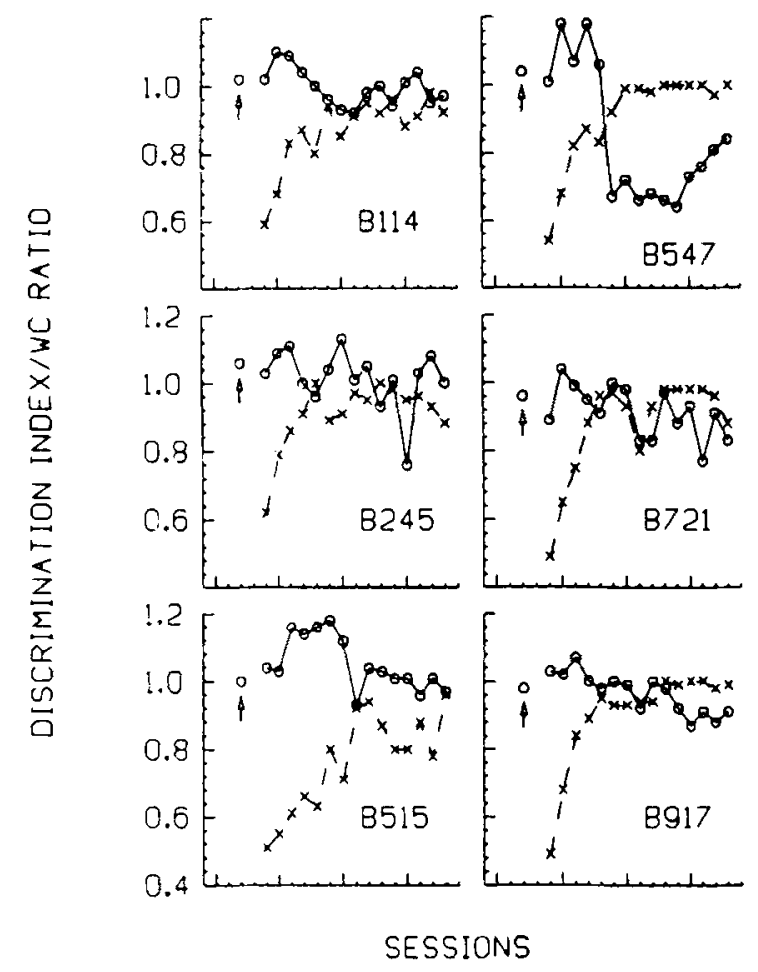

Figure 4. Changes in the discrimination index (plotted as Xs) and the whithin-component (WC) ratio (plotted as Os) during the first testing (RI/Ext) condition, when schedules strictly alternated. See captions to Figures 1 and 2 for information about these measures. Dath are shown in separate panels for all binds in Group 3, associnted with the largest stimulus difference. Birds 515 and 721 were the experimentally naive subjects. Arrows highlight the leftmost points, which show the mean WC ratio during the preceding baseline condition. The remaining points describe means taken over single testing seasions.

Although the present work defines more precisely the relationship between local contrast and discriminability, this association is still not fully understood. The decline associated with very poor discriminations can be attributed to stimulus generalization; however, the intriguing question concerns the decline with improving discrimination. In the past decade, analyses of local contrast have invoked Pavlovian conditioning because the responses in question seem to be autoshaped (Schwartz, 1978). Indeed, Pavlov (1927) described an allied effect: The magnitude of the conditioned response was larger immediately following a negative stimulus, especially when the discrimination was poorly learned. He attributed the effect to a transient inhibitory aftereffect. Related explanations have been advanced by Mackintosh (1974) and Malone and Staddon (1973). However, further empirical support is needed for the thesis that such rebound effects are strengthened by partial generalization between inhibitory and excitatory stimuli.

Earlier and seemingly related approaches invoke the frustrative effects of extinction (Terrace, 1966) or punishment (Bloomfield, 1966) and perhaps have been too severely discredited. A study frequently invoked to discon- firm this approach is that of Kodera and Rilling (1976). Their work showed a poor association between extinction responding and overall contrast, but it did not isolate local contrast. The present data support those of Bloomfield (1966), demonstrating a positive relation between extinction responding and transitory ("peak") contrast; Figure 3 (right) replicates Bloomfield's finding for the between-component measure, which emphasizes schedule transitions. For the within-component measure, a close ally of extinction rate, the DI, accounts for more of the variance. This outcome and the questions raised here about the importance of sequential factors suggest that positive local contrast is favored by a general context that supports uncertainty about component values. For example, enhanced responding at the onset of new components may constitute observing behavior, which would intensify exposure to poorly defined visual stimuli. It is important to note, however, that explanations invoking frustration and uncertainty do not explain the relation between discrimination and negative local contrast (P. $M$. Blough, 1983).

Additional findings from this study address Williams's (1983) analysis, which distinguishes between the variables that affect local and overall contrast. According to Williams, local contrast depends on the value of the preceding component; this explanation invokes the sequential effects discussed above. Overall contrast, according to Williams's analysis, depends on the value of the following component, which the subject learns to anticipate. This explanation rests on evidence that the direction of steadystate contrast depends on the schedule during the following, rather than the preceding, component; for example, rates in a constant component are higher when the following schedule is lean than when it is rich (Buck et al., 1975; Williams, 1979, 1981, 1982; Williams \& Wixted, 1986). Since following-schedule control is acquired with training, it appears and persists as local contrast diminishes.

To apply Williams's (1983) analysis to the present data, it is helpful to consider the cues that would aid followingschedule control. When multiple-schedules components strictly alternate, temporal or sequential information could be useful. In addition, cues to the current schedule may strengthen the sequential information; that is, this information might be more useful when the identity of the current schedule is clear. Thus, following-schedule control should be most marked when current-component and sequential cues are strong. Indeed, this prediction is born out in the alternating condition; for birds that discriminated well, overall contrast persisted and the local form dissipated (cf. Figures 1 and 2, top panels; see also Figure 4). For birds that discriminated less well, both local contrast and overall contrast persisted, perhaps because the sequential information was degraded and preceding-component effects retained control of both forms of contrast. The present data are not entirely consistent with Williams's analysis, however. Since a higher weight is assigned to following-schedule control, overall 
contrast should have declined in the random condition. It did not (see Table 2).

Also relevant to Williams's (1983) analysis is a comparison of within-component patterns for the two testing conditions. In the random condition, the reduction of sequential information should have led to stronger precedingschedule control and enhancement of local contrast. If weak stimulus cues further diminish following-schedule information, the difference should have been more marked when discriminability was poorer. The data are consistent with this prediction; that is, within-component ratios increased following the introduction of the random condition, and this effect was more marked in Groups 1 and 2 (see Table 3 and associated text).

Contrast studies in this laboratory have always required short ITIs. It could be argued that these time-outs modified relationships between components. For example, the ITI could have been a predictable following schedule in the random condition, and thus have sustained overall contrast. However, overall contrast was assessed with reference to baseline conditions that also included the ITI; thus, the measure controlled for any such effect. Similar logic could suggest that the ITI constituted a "preceding schedule" that led to local contrast in components following equally valued schedules. It is possible that the 2 -sec ITI used here contributed to instances of local contrast during baseline and lessened the effectiveness of betweengroup comparisons. However, if ITIs were important, they would have overshadowed the discriminability relationships seen here and in our previous work (e.g., D. S. Blough, 1975; P. M. Blough, 1983; P. M. Blough \& D. S. Blough, 1985). Finally, P. M. Blough (1983) also showed negative local contrast, which should not follow a controlling ITI. In sum, the ITI almost certainly did not contribute to this study's major finding, the functional relation between local contrast and discrimination performance.

\section{REFERENCES}

Bloomfield, T. M. (1966). Two types of behavioral contrast in dis crimination learning. Joumal of the Experimental Analysis of Behavior, 9, 155-161.

BLough, D. S. (1957). Spectral sensitivity in the pigeon. Journal of the Optical Society of America, 47, 827-833.

BLougH, D. S. (1975). Steady state data and a quantitative model of operant generalization and discrimination. Joumal of Experimental Psychology: Animal Behavior Processes, 1, 3-21.

BLough, P. M. (1983). Local contrast in multiple schedules: The effect of stimulus discriminability. Journal of the Experimental Analysis of Behavior, 39, 427-435.

Blough, P. M., \& Blough, D. S. (1985). Sequential effects in dimensional contrast. Journal of the Experimental Analysis of Behavior, 44 233-244.

Buck, S., Rothstein, B., \& Willams, B. A. (1975). A re-examination of local contrast in multiple schedules. Journal of the Experimental Analysis of Behavior, 24, 291-301.

Catania, A. C., \& Gill, C. A. (1964). Inhibition and behavioral contrast. Psychonomic Science, 1, 257-258.
FreUnd, R. J., L LTTELL, R. C. (1981). SAS for linear models: A guide to the ANOVA and GLM procedures. Cary, NC: SAS Institute.

HerRnstein, R. J. (1970). On the law of effect. Joumal of the Experimental Analysis of Behavior, 13, 243-266.

Kodera, T. L., Rilling, M. (1976). Procedural antecedents of behavioral contrast: A re-examination of errorless learning. Journal of the Experimental Analysis of Behavior, 25, 27-42.

MACKINTOSH, N. J. (1974). The psychology of animal learning. London: Academic Press.

Mackintosh, N. J., LitTle, L., \& LoRD, J. (1972). Some determinants of behavioral contrast in pigeons and rats. Learning \& Motivation, 3, 148-161.

MALONe, J. C., JR. (1976). Local contrast and Pavlovian induction. Journal of the Experimental Analysis of Behavior, 26, 425-440.

MALONE, J. C., \& RowE, D. W. (1981). Local contrast, local dimensional effects, and dimensional contrast. In M. L. Commons \& J. A Nevin (Eds.), Quantitative analyses of behavior: Vol. 1. Discriminative properties of reinforcement schedules (pp. 365-390). Cambridge, MA: Ballinger.

Malone, J. C., JR., \& Staddon, J. E. R. (1973). Contrast effects in maintained generalization gradients. Joumal of the Experimental Analysis of Behavior, 9, 305-315.

MCSWeEney, F. K., Norman, W. D. (1979). Defining behavioral contrast for multiple schedules. Joumal of the Experimental Analysis of Behavior, 32, 457-461.

Nevin, J. A., \& Shettleworth, S. J. (1966). An analysis of contrast effects in multiple schedules. Journal of the Experimental Analysis of Behavior, 9, 305-315.

Pavlov, I. P. (1927). Conditioned reflexes: An investigation of the physiological activity of the cerebral cortex (Anrep translation). New York: Dover.

SCHWARTZ, B. (1978). Stimulus-reinforcer contingencies and local behavioral contrast. Joumal of the Experimental Analysis of Behavior 29, 297-308.

Spealman, R. D., Gollub, L. R. (1974). Behavioral interactions in multiple variable-interval schedules. Journal of the Experimental Analysis of Behavior, 22, 471-481.

TERRACE, H. S. (1966). Behavioral contrast and the peak shift: Effects of extended discrimination training. Joumal of the Experimental Analysis of Behavior, 9, 613-617.

White, K. G., PIPE, M., \& McLean, A. P. (1984). Stimulus and reinforcer relativity in multiple schedules: Local and dimensional effects on sensitivity to reinforcement. Journal of the Experimental Analysis of Behavior, 41, 69-81.

Williams, B. A. (1979). Contrast, component duration, and the following schedule of reinforcement. Journal of Experimental Psychology: Animal Behavior Processes, 5, 379-396.

Williams, B. A. (1981). The following schedule of reinforcement as a fundamental determinant of steady state contrast in multiple schedules. Journal of the Experimental Analysis of Behavior, 35, 293-310.

WiLliams, B. A. (1982). Do interactions in multiple and concurrent schedules have a common basis? In M. L. Commons, R. J. Herrnstein, \& H. Rachlin (Eds.), Quantitative analyses of behavior: Matching and maximizing accounts (Vol. 2, pp. 281-302). Cambridge, MA: Ballinger.

Williams, B. A. (1983). Another look at contrast in multiple schedules. Journal of the Experimental Analysis of Behavior, 39, 345-384.

Williams, B. A. (1988). The effects of stimulus similarity on different types of behavioral contrast. Animal Learning \& Behavior, 16, 206-216.

Williams, B. A., \&ixted, J. T. (1986). An equation for behavioral contrast. Journal of the Experimental Analysis of Behavior, 45, 47-62.

(Manuscript received February 16, 1988; revision accepted for publication June 8,1988 .) 